\title{
Purple Urine Bag Syndrome in Geriatric Hospital: A Report of 2 Cases
}

\author{
Kyung Su Shin ${ }^{1}$, Mi Young Kim², Hyo Nam Kang ${ }^{2}$ \\ ${ }^{1}$ Department of General Surgery, Yeosunoble Geriatric Hospital, Yeosu, Korea \\ ${ }^{2}$ Department of Nursing, Yeosunoble Geriatric Hospital, Yeosu, Korea
}

Corresponding Author:

Kyung Su Shin

https://orcid.org/0000-0002-3724-3538

Department of General Surgery,

Yeosunoble Geriatric Hospital, 40,

Heungguk-ro, Yeosu 59676, Korea

Tel: +82-61-688-0000

Fax: +82-61-686-0193

E-mail: prid3614@naver.com

Received: August 20, 2018

Revised: August 29, 2018

Accepted: August 30, 2018
Purple urine bag syndrome (PUBS) is a rare clinical condition that occurs in older women with a long-term urinary catheterization. Other risk factors include alkaline urine, constipation, renal disease, and immobility. It presents as purple discoloration of the urine bag and tubing and is a mixture of pigment that becomes purple. Patient with PUBS usually do not complain of any symptoms. The clinical course is generally benign, and intensive treatment is not usually needed. But awareness of this syndrome is necessary for doctor and nurse in geriatric hospital so as to adequately treat urinary tract infection and explain the clinical process to a caregiver. We present 2 cases of PUBS in a geriatric hospital. (Ann Geriatr Med Res 2018;22:151-153)

Key Words: Elderly female, Purple discoloration, Purple urine bag syndrome

\section{INTRODUCTION}

Purple urine bag syndrome (PUBS) is a rare syndrome associated with alkaline urine and sometimes urinary infections that are frequently observed in elderly women with long-term catheterization. ${ }^{1,2)}$ According to the national population statistics ${ }^{3)}$ published by the Ministry of the Interior and Safety of Korea at the end of 2017, 14.2\% of the population was older than 65 years; thus, Korea is becoming a full-fledged senior society. The Korean Statistical Information Service ${ }^{4)}$ also reported 1,535 geriatric hospitals nationwide in the first quarter of 2018. With the increasing number of elderly patients who have been hospitalized for a long time in Korea, it is estimated that the actual prevalence of PUBS will be much higher. ${ }^{5)}$ We describe 2 patients with PUBS, which has not been commonly reported in Korea.5. They had no clinical symptoms or signs of urinary or systemic infection, so we observed a benign clinical course of PUBS that did not require antibiotics.

\section{CASE REPORTS}

\section{Case 1}

An 81-year-old bedridden woman was hospitalized because of loss of appetite and nausea. She had been hospitalized in nearby hospitals for a peptic ulcer and heart failure 2 months ago. She had diabetes, hypertension, and congestive heart failure for 10 years and was unable to walk for 3 months before admission. Her surgical and family histories were unremarkable.

She received a diuretic (furosemide, $40 \mathrm{mg}$ ), an oral antidiabetic drug (metformin, 1,000 mg), a neurotonic drug (donepezil, $5 \mathrm{mg}$ ), and laxative (magnesium hydroxide, $1,000 \mathrm{mg}$ ) for chronic constipation. Additionally, a stimulant laxative (bisacodyl, $10 \mathrm{mg}$ ) was frequently administered.

Uncontrolled leakage of urine caused the patient distress and decreased her quality of life. Thus, after consulting with the patient's family, we inserted a Foley catheter to improve her quality of life and did not perform further evaluation of urinary incontinency. The urine bag we used was made of medical grade polyvinyl chloride, and the catheter was made of 100\% silicon.

The catheter we used was in accordance with the Korea Centers for Disease Control and Prevention standards for 2017. The catheter and urine bag were not regularly replaced unless the catheter was blocked, closed urinary system was broken, connection was disconnected, or urine leaked. Skin disinfectants were not used to disinfect around the urinary tract. The bulb of the urethra was cleaned during routine hygiene practices, such as showering or bathing. The urine bag was not filled more than $3 / 4$ full, and it was emptied 3 times per day at 8-hour intervals.

The patient was hospitalized for 14 months, and purple urine was first observed at 9 months during her hospitalization. After changing the catheter, purple urine was observed again about 8 or 10 days later, and it changed to a darker purple color over time (Fig. 1A). Subsequently, 

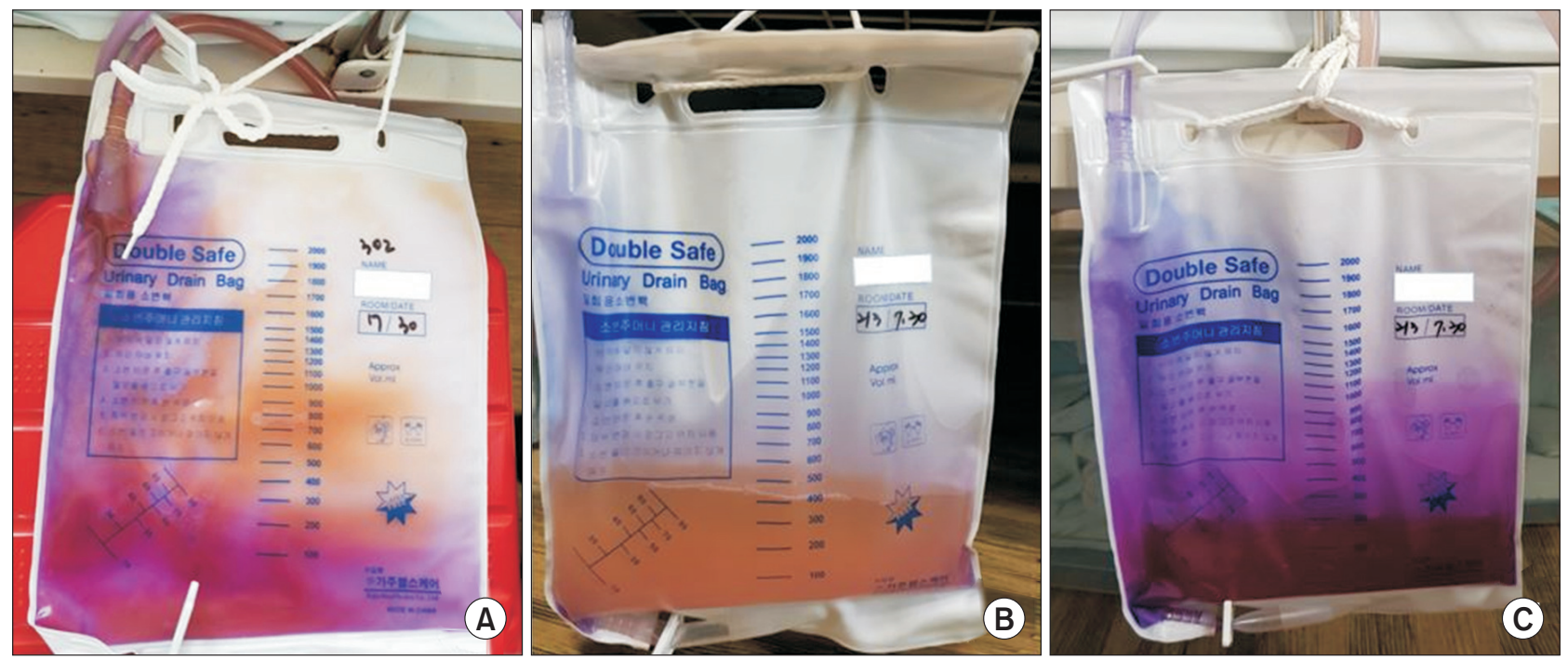

Fig. 1. Purple color of the urine bag and tube. (A) Purple-colored urine in case 1. (B) In case 2, about 5 days later after changing the catheter. (C) In case 2, darker purple over time.

PUBS lasted 5 more months and then resolved. The only condition that changed was that the patient's bean curd intake decreased.

When the purple urine was observed, there were no infectious symptoms, such as chills or fever. In addition, the laboratory findings, peripheral blood test results, liver function test results, and electrolyte test results were all normal. The creatinine level was within normal range $(1.2 \mathrm{mg} / \mathrm{dL})$. Results of the urinalysis showed the following: urine specific gravity, 1.015; pH level, 8.0; protein, traceable; and nitrite, $1+$. In the microscopic examination of urine sediment, the red blood cell count was 3-5/high power field (HPF), white blood cell count was 3-5/HPF, and presence of bacteria was negative.

As there were no clinical signs and symptoms of a urinary tract infection, a bacterial culture was not performed, and antibiotic therapy was not provided.

\section{Case 2}

An 88-year-old bedridden woman underwent percutaneous coronary angioplasty 2 weeks before admission, and until she was stabilized, she was hospitalized for observation in other hospitals. Her medical history also included hypertension, and her surgical and family histories were unremarkable.

The patient received antithrombotic agents (clopidogrel, $75 \mathrm{mg}$ and aspirin, $100 \mathrm{mg}$ ), an antihypertensive drug (losartan potassium, $50 \mathrm{mg}$ ), a diuretic (furosemide, 40 $\mathrm{mg}$ ), vasodilator (nicorandil, $5 \mathrm{mg}$ ), and neurotonic drug (donepezil, $5 \mathrm{mg}$ ). She did not take any constipationrelated medications.

A Foley catheter was inserted because of severe skin impairment of the perineum and buttocks due to frequent leakage of urine. She had a stage 3 pressure ulcer on her coccyx.
The patient was hospitalized for 11 months, and purple urine was first observed at 3 months during her hospitalization. After changing the catheter, purple urine was observed again about 5 days later (Fig. 1B). As in the first case, the color of the urine became dark purple over time (Fig. 1C). PUBS persisted until the patient was discharged. We could not perform further follow-up thereafter.

This patient also did not show any symptoms of infection. The laboratory findings were all normal, and the creatinine level was within normal range $(0.8 \mathrm{mg} / \mathrm{dL})$. A bacterial culture was not performed, and antibiotic therapy was not provided.

\section{DISCUSSION}

PUBS was first reported in 1978 by Barlow and Dickson (quoted from ${ }^{1,6}$ ). This syndrome is an uncommon condition characterized by purple urine in the urine bag and tubing in elderly women with prolonged urinary catheterization. $^{6-9)}$

Proposed risk factors are a long-term indwelling urinary catheter, female sex, older age, and the presence of chronic constipation, chronic renal disease, bedridden state, alkaline urine, and urinary tract infection. ${ }^{1,5,10,11)}$

The pathogenesis of PUBS is summarized in Fig. 2. PUBS is due to the metabolism of tryptophan by intestinal bacteria indole and conversion to indoxyl sulfate (indican) by conjugation in the liver. This is secreted into urine, and then indoxyl sulfate is catalyzed by bacterial phosphatases or sulfatases to indoxyl in the urinary tract. In alkaline urine, indoxyl is converted into 2 pigments: indigo (a blue pigment) and indirubin (a red pigment). These pigments mix and react with the urine bag and tubing and produce a purple-colored urine. ${ }^{1,5,11-13)}$

Several bacteria are often associated with PUBS. These 


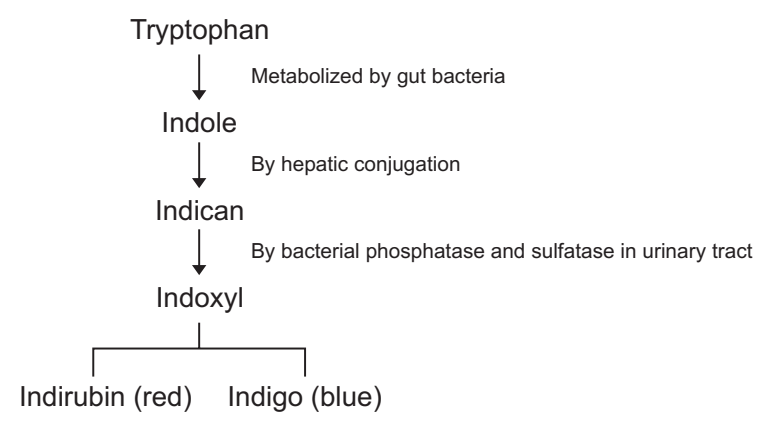

Fig. 2. Pathogenesis of purple urine bag sydrome.

bacteria are mainly gram negative and include Providencia rettgeri, Providencia stuartii, Proteus mirabilis, Klebsiella pneumonia, Enterococcus species, Escherichia coli, Morganella morganii, Pseudomonas aeruginosa, Citrobacter species, and group B streptococci. It is assumed that intestinal bacteria are involved in the pathogenesis of PUBS. ${ }^{1,5,10,11)}$

Two studies ${ }^{5,14)}$ reported that patients with the syndrome had a benign clinical process. However, the occurrence of PUBS can be confusing to patients, their families, caregivers, and even inexperienced medical staff. This confusion can cause anxiety for the patients and their families, and the physician may make a misdiagnosis. After acquiring knowledge of the risk factors and pathogenesis of this syndrome, physicians and nurses in a geriatric hospital will be able to provide a sufficient explanation of PUBS to patients, and the medical staff will avoid making a misdiagnosis or providing improper treatment, such as an overdose. These processes will help establish a positive relationship between patients and the medical staff.

Shiao et al. ${ }^{15)}$ and $\mathrm{Ga}^{14)}$ recommended improving patient's hygiene and changing the catheter on a regular basis as in other patients with catheters. However, the authors only replaced the catheter if the patient had severe anxiety or the urine bag and tubing were unsanitary.

Kim et al. ${ }^{5)}$ suggested that without symptoms and signs of urinary tract infection, the use of preventive antibiotics is not recommended because of the possibility of antibiotic-resistant bacteria. $\mathrm{Ga}^{14)}$ suggested that PUBS was unrelated to the progression of a urinary tract infection or sepsis so it is inappropriate to administer antibiotics to elderly patients with impaired immune systems.

The clinical outcomes of PUBS are not always benign, and many authors have reported patients who have died of severe infections, such as septic shock or aspiration pneumonia. ${ }^{14,15)}$ According to a recent study that analyzed 106 articles (174 cases), which investigated PUBS and were indexed in PubMed from 1980 to 2016, the death rate was $6.8 \%{ }^{10)}$

In conclusion, if purple urine appears in a long-term patient in a geriatric hospital, physicians and nurses should first provide a full explanation to the patient and their families about PUBS.,111 Then it would be reasonable for the physician to closely examine the patient's clinical condition, eliminate modifiable risk factors, make an early diagnosis of PUBS, and provide treatment immediately when the syndrome transitions to infection. ${ }^{10)}$ Even if patients with PUBS do not have symptoms of infection, we recommend that the physician perform further evaluation to determine the cause, if possible.

\section{CONFLICTS OF INTEREST DISCLOSURES}

The researchers claim no conflicts of interest.

\section{REFERENCES}

1. Agbor VN, Njim T, Oben HB, Aminde LN. Purple urine bag syndrome in an 80-year-old Female Cameroonian. Niger Med J 2017; 58:119-21.

2. Kalsi DS, Ward J, Lee R, Handa A. Purple urine bag syndrome: a rare spot diagnosis. Dis Markers 2017;2017:9131872.

3. MOIS: the population of Korea at the end of 2017 as press release [Internet]. Sejong (Korea): Ministry of the Interior and Safety; [cited 2018 Jan 9]. Available from: http://www.mois.go.kr.

4. KOSTAT: the medical institution of Korea [Internet]. Daejeon (Korea): Korean Statistical Information Service; [cited 2018 May 25]. Available from: http://kostat.go.kr.

5. Kim JH, Jung JS, Kim MJ. Purple urine bag syndrome. J Korean Geriatr Soc 2012;16:221-4.

6. Stott A, Khan M, Roberts C, Galpin IJ. Purple urine bag syndrome. Ann Clin Biochem 1987;24(Pt 2):185-8.

7. Dealler SF, Hawkey PM, Millar MR. Enzymatic degradation of urinary indoxyl sulfate by Providencia stuartii and Klebsiella pneumoniae causes the purple urine bag syndrome. J Clin Microbiol 1988;26:2152-6.

8. Al-Jubouri MA, Vardhan MS. A case of purple urine bag syndrome associated with Providencia rettgeri. J Clin Pathol 2001;54: 412.

9. Lin HH, Li SJ, Su KB. Purple urine bag syndrome: a case report and review of the literature. J Intern Med Taiwan 2002;13:209-12.

10. Yang HW, Su YJ. Trends in the epidemiology of purple urine bag syndrome: a systematic review. Biomed Rep 2018;8:249-56.

11. Ga H. Purple urine bag syndrome. J Korean Geriatr Soc 2008;12:14.

12. Lin CH, Huang HT, Chien CC, Tzeng DS, Lung FW. Purple urine bag syndrome in nursing homes: ten elderly case reports and a literature review. Clin Interv Aging 2008;3:729-34.

13. Lin J, Hlafka M, Vargas O, Bhattarai M. Recurrent purple urine bag syndrome presenting with full spectrum of disease severity: case report and review of literature. CEN Case Rep 2016;5:144-7.

14. Ga H. Purple diaper syndrome self remitted without antibiotic treatment. Geriatr Gerontol Int 2012;12:571-2.

15. Shiao CC, Weng CY, Chuang JC, Huang MS, Chen ZY. Purple urine bag syndrome: a community-based study and literature review. Nephrology (Carlton) 2008;13:554-9. 